\title{
Polyphenols slash the risk of cancers: a mini review
}

\begin{abstract}
Polyphenols are auxiliary metabolites of plants and are for the most part engaged in providing protection by acting as barrier against pathogens. More than 500 diverse polyphenols exist and are arranged their bioavailability varies in according to their site of retention in human beings. Phenolic compounds are present in plants at different levels, as the external layers of plants contain more amounts of phenolic compounds than those situated in their inward parts. Numerous experimental studies exhibited the role of naturally occurring polyphenols in fruits and vegetables due to their beneficial effects on human body and giving the significant protection against many cancers. Polyphenols containing many properties such as, anti-oxidative, anti-proliferative, anti-metastatic, anti-inhibitory and anti-carcinogenesis. Chief role in the activation of guardian gene $\mathrm{p} 53$ in human body and suppression of reactive oxygen species. Current review summarized the anticancer potential of polyphenols.
\end{abstract}

Volume 6 Issue 6 - 2018

\author{
Fasiha Ahsan,' Muhammad Imran,' Shahid \\ Bashir,' Syed Amir Gilani,' Awais Raza,' \\ Muhammad Hanif Mughal ${ }^{2}$ \\ 'University Institute of Diet and Nutritional Sciences, University \\ of Lahore, Pakistan \\ ${ }^{2}$ Department of Chemistry, University of Swabi, Pakistan
}

\begin{abstract}
Correspondence: Muhammad Imran, University Institute of Diet and Nutritional Sciences, University of Lahore, Pakistan, Tel: +923324746613, Email mik_1661@yahoo.com
\end{abstract}

Received: October 10, 2018 | Published: November 14, 2018

Keywords: polyphenols, anti-cancer, fruits and vegetables, flavonoids, p53 gene

\section{Introduction}

Cancer is a generic term for a large group of disorders that can affect any part of the body. It occurs mostly because of alterations in genes which control or regulate the normal function of the cells. Cancer is the rapid creation of the abnormal cell growth beyond their usual boundaries and spread to other parts of the body. A tumor can be benign or malignant. A benign tumor is not considered cancerous whereas malignant tumor is considered dangerous due to numerous mitotic activities and it spread to their surrounding and other tissues of the body. ${ }^{1}$ Cancer is the leading cause of death worldwide accounting for 8.2 million deaths by $2017 .{ }^{2}$ According to estimation 33 million people are diagnosed with cancer within the previous five years. Most were women with the diagnosis of breast cancer ( 6.3 million), men with prostate cancer (3.9 million) and diagnosis of colorectal cancer in both men and women were 3.5 million. ${ }^{3}$ The most commonly prevalent cancers with their death rates are; Lung (1.69million deaths), Liver (789000 deaths), colorectal (774000 deaths), stomach (755000 deaths) and breast (572000 deaths). ${ }^{3}$ It is estimated that there are 150000 new cancer cases reported/year in Pakistan and 60 to $80 \%$ of these patients were died each year by diagnosed cancer. ${ }^{4}$ The age standardization for cancers are 172/100000 for females and 145/100000 for males. Top ten cancers (Table 1) in males are; Lung (11\%), Lip and oral cavity $(10.5 \%)$, Stomach $(7 \%)$ other pharynx $(5.6 \%)$, leukemia $(5.2 \%)$, colorectal (6.9\%), esophagus (5.4\%), bladder (4.6\%), Larynx (4.3\%) other unspecified (39.5\%). Top ten cancers in females are; breast (26\%), Lip and oral cavity (6.6\%), ovary $(4.8 \%)$, esophagus $(4.2 \%)$, colorectal (3.3\%), cervix/uterine (15.7\%), stomach (3.4\%), leukemia (3.3\%), Non-Hodgkin's lymphoma $(2.4 \%)$ other and unspecified $(30.3 \%) .{ }^{4}$ The purpose of this mini review to highlights the potential of polyphenols in the prevention of different cancers due to the presence of specialized compounds in their structures.

Polyphenols are normally discovered to a great extent due to their advantages on human body to greater extent. Mainly present in the natural products, vegetables, grains and refreshments. Organic products like grapes, apple, pear, fruits and Berries contains up to $200-300 \mathrm{mg}$ polyphenols per $100 \mathrm{gram}$ of weight. The items made from these natural products, additionally contain polyphenols in critical sums. Likewise, oats, dry vegetables and chocolate also add for polyphenol consumption. ${ }^{5}$ Polyphenols are auxiliary metabolites of plants and are for the most part engaged in providing protection by acting as barrier against pathogens. Researchers have investigated that these particles are great cell reinforcements and may kill the damaging reactivity of undesired responsive oxygen/nitrogen species delivered as by item during metabolic procedures in the body. Epidemiological examinations have covered that polyphenols give a critical assurance against protection of few unending ailments for example, tumor, cardiovascular ailments, diabetes, growth maturing and asthma. ${ }^{6,7}$

Table I Top ten cancers in males and females ${ }^{4}$

\begin{tabular}{llll}
\hline \multicolumn{2}{l}{ Top I 0 cancers in males } & \multicolumn{2}{l}{ Top I0 cancers in females } \\
\hline Cancers & Percentages (\%) & Cancers & Percentages (\%) \\
\hline Lung & II & Breast & 26 \\
Lip/oral & 10.5 & Lip/oral & 6.6 \\
Stomach & 7 & Ovary & 4.8 \\
Pharynx & 5.6 & Esophagus & 4.2 \\
Esophagus & 5.4 & Stomach & 3.4 \\
Leukemia & 5.2 & Leukemia & 3.3 \\
Colorectal & 6.9 & Colorectal & 3.3 \\
Bladder & 4.6 & Cervix uterine & 15.7 \\
Larynx & 4.3 & Non-Hodgkin's & 2.4 \\
Unspecified & 39.5 & Unspecified & 30.3 \\
\hline
\end{tabular}

\section{Classification and occurrence of polyphenols}

More than 500 diverse polyphenols exist and are arranged in light of structure having hydroxyl group in their rings and considered as supporting elements for functioning of different organs. Differences in essential fragrant rings, oxidation status, and useful gatherings. Some examples are: flavonoids, lignans, stilbene and phenolic compounds. ${ }^{8}$ Flavonoids are made out of a three ring structure and can be subdivided by the proximity of an oxygen gather at position 4 , a two-fold bond between carbon items 2 and 3, or a hydroxyl bunch in positive 3 of 
the $\mathrm{C}$ (center) ring. ${ }^{9}$ This class is partitioned into the accompanying essential epigallocatechin, epicatechingallate, epigallocatechingallate and procyanidin. Anthocyanin class of polyphenols fundamentally comprise of anthocyanidin with various sub classes: flavonols, flavanones, flavan-3-ols, flavones, anthocyanin and isoflavones. Flavonols including kaempferol, myricetin and quercetin are prevalent and are found in citrus organic products. Some others are maringenin and hesperetin. Flavan-3-ols containing epicatechin, gallocatechin and anthocyanin which are obvious in quantity and having red/purple shading e.g. berries and red wine. Isoflavones are one of important kind of polyphenols that look like estrogen in structure and are named as phytoestrogens; these substances are found in soy items such a tofu, cooked soy nuts and miso. Another class of polyphenols is lignans, which are described by their 1,4-di-arylbutana structure and are found in the most of (lariciresinol, matairesinol) in seeds e.g. flax and sesame seeds. ${ }^{5}$ The essential polyphenols in the phenolic corrosive class are hydroxybenzoic corrosive and hydroxycinnamic corrosive and can be found in calculable amounts in espresso, walnuts, plums and blueberries. The stilbene class is also essential and characterized by resveratrols, 1,2-diarylethene structure which are found in red wine and thought to contain calming properties. ${ }^{10}$

Phenolics compounds are present in plants at different levels like tissue, cell and sub-cell. Insoluble phenolics are found in cell dividers, while dissolvable phenolics are available inside the plant cell vacuoles. The external layers of plants contain more elevated amounts of phenolics than those situated in their inward parts. Various variables influence the polyphenol substance of plants, these incorporate level of readiness at the season of reap ${ }^{7}$. Polyphenolic substances of nourishments are greatly influenced by natural factors and also by the factors like soil, sun exposure and precipitation. Another factor that straightforwardly influences the polyphenol substance of the nourishments is change on capacity, the reason is simple which is oxidation of polyphenols. ${ }^{7}$ Oxidation responses result in the arrangement of pretty much polymerized substances, which prompt changes in the nature of nourishments especially in shading. Such change might be useful, just like the case with dark tea or carmelizing of natural product. Onions and tomatoes lose in the vicinity of $75 \%$ and $80 \%$ of their underlying quercetin content subsequent to bubbling for $15 \mathrm{~min}, 65 \%$ in the wake of cooking in a microwave stove and $30 \%$ in the wake of frying. ${ }^{11}$

\section{Bioavailability of polyphenols}

Bioavailability is the extent of the supplement that is processed, assimilated and used through ordinary pathways. ${ }^{7}$ Bioavailability of every single polyphenol contrasts however there is no connection between the amounts in human body. Most polyphenols are available as esters, glycosides or polymers that cannot be caught up in local frame. Before retention, polyphenols experience broad adjustment in certainty they are conjugated in the intestinal cells and later in the liver by methylation, sulfation or potentially glucuronidation. ${ }^{7}$ Polyphenols bioavailability varies in according to their site of retention in human beings. A portion of polyphenols are very much retained in the gastro-intestinal tract while others in digestive system or in piece of the stomach. All flavonoids with the exception of flavanols exist in glycosylated shapes. The destiny of glycosides in the stomach isn't clear yet. Most of the glycosides presumably oppose corrosive hydrolysis in the stomach and along these lines arrive in digestive tract. ${ }^{12}$ Study demonstrated that the assimilation at gastric level is acceptable for a few flavonoids e.g. quercetin. Moreover it has been also demonstrated that, in rats and mice anthocyanins are consumed from the stomach. Assessment of dietary polyphenols consumption has been proposed through measurement in serum and metabolites. ${ }^{13}$

\section{Experimental studies of polyphenols and cancer prevention}

Resveratrol is chiefly present grapes, red wine and raspberries. Different studies demonstrated that 15-20 micro-molar of resveratrol intake considerably reduce the proliferation of cancer cells in lungs. ${ }^{14,15}$ It also involves in the prevention of gastro-intestinal tract (GIT) cancer in 30-40 micro-molar concentration. ${ }^{15}$ However, at very high dosage like 55 to 250 micro-molar, resveratrol prompted DNA damage and cell death in patients with stomach carcinoma by increasing the generation of reactive oxygen species. ${ }^{16}$ Another study results exhibited that supplements of resveratrol equal to $0.1-0.2 \mathrm{~g}$ / day for human beings provides protection against colorectal cancer by conquering the pathway of Kras. ${ }^{17}$ Although its bioavailability is low but its benefits against cancer protection are well documented. ${ }^{18}$ Provision of resveratrol at initial stages of hepatic cancer was also effective because it suppresses the metastasis. ${ }^{19}$ Naringenin polyphenol provide protection against liver cancer by arresting the cell cycle from $\mathrm{G}$ phase enhanced the activation of $\mathrm{p} 53$ gene..$^{20}$ Colon cancer inhibition also demonstrated by different studies which exhibited basic mechanism that is the enhancement of apoptotic activity mediated by $\mathrm{p} 38$ gene. ${ }^{21}$ Lignans are structurally analogous to estradiol that's why have anticancer potential especially for hormonal cancers such as breast, prostate and colon. Sesame oil in 10-40 micromolar in concentration positively enhanced the apoptosis and reduced the rate of vascular endothelial growth factor, macrophages and viability of cells. ${ }^{22}$ Quercetin is another polyphenol with potential role in cancer inhibition. Researchers found that when $49 \mathrm{mg} / \mathrm{kg} /$ day were given to the patients of colon cancer then it reduced the proliferation of cancer cells in specific area by increasing apoptosis. ${ }^{23}$ Its role in breast cancer reduction is also very admirable, its 100-200micro-molar concentration effectively induced the apoptosis and down regulate the activity of breast cancer biomarkers Bcl-2. ${ }^{24}$ Breast cancer in females also suppressed by giving $30 \mathrm{mg} / \mathrm{kg} /$ day dosage of quercetin. Basically, it acts on the vascular endothelial growth factor pathway and decreased the rate of angiogenesis in mice. ${ }^{25}$ In male rat models prostate cancer also be inhibited by using $220 \mathrm{mg} / \mathrm{kg} /$ day of quercetin for twice a week. ${ }^{26}$ Polyphenol kaempferol also has positive role in human body by its unique properties including allergic prevention, diabetes suppression and cardio-protective. ${ }^{27}$ Study indicated that kaempferol involves in stomach cancer suppression by arrest the mitotic phase of cell cycle and induced the cell death of abnormal cells in stomach area. ${ }^{28}$ Breast cancer cells also reduced in females by receiving the treatment with kaempferol (90-100micro-molar in concentration), basic mechanism in this reduction is inhibition of glucose uptake by glucose transporter-1 (GLUT-1) receptors which further relate to anti-proliferation. ${ }^{29}$ It also down regulates the mitogen activated protein kinase (MAP-K) pathway and further reduced the incidence of breast cancer. ${ }^{30}$ Health perspectives of polyphenols are summarized in Table 2. 
Table 2 Health perspectives of polyphenols

\begin{tabular}{|c|c|c|c|c|}
\hline Compounds & Dietary sources & Biological effects & Cancer prevention & References \\
\hline Quercetin & $\begin{array}{l}\text { Apricot, onions, berries, nuts, seed, tea, } \\
\text { broccoli, apples }\end{array}$ & $\begin{array}{l}\text { Tumor suppression } \\
\text { Antiviral } \\
\text { Anti-oxidative }\end{array}$ & $\begin{array}{l}\text { Gastric, } \\
\text { Breast, Liver, }\end{array}$ & Duo et al. ${ }^{24} \&$ Zhao et al. ${ }^{25}$ \\
\hline Kaempferol & $\begin{array}{l}\text { Peaches, black berries, apples, grapes, } \\
\text { tomatoes, green tea }\end{array}$ & $\begin{array}{l}\text { Suppress lipid } \\
\text { oxidation }\end{array}$ & $\begin{array}{l}\text { Prostate, Colorectal } \\
\text { and Lung Cancers }\end{array}$ & Jo et al. ${ }^{27}$, Song et al. ${ }^{28} \& \mathrm{Li}$ et al. ${ }^{30}$ \\
\hline \multirow{3}{*}{ Naringenin } & \multirow{3}{*}{ Oranges, grape fruit, tomatoes } & $\begin{array}{l}\text { Protection against } \\
\text { bacterial and fungal } \\
\text { reactions }\end{array}$ & & \multirow{3}{*}{$\begin{array}{l}\text { Arul Subramanian }{ }^{20} \& \text { Song et } \\
\text { al.21 }\end{array}$} \\
\hline & & $\begin{array}{l}\text { Decrease the } \\
\text { propagation of } \\
\text { platelets }\end{array}$ & & \\
\hline & & $\begin{array}{l}\text { Suppress the activity } \\
\text { of VEGF } \\
\text { Inhibit the formation } \\
\text { of ROS }\end{array}$ & & \\
\hline Resveratrol & Blueberries, raspberries, grapes & & & $\begin{array}{l}\text { Wang et al. }{ }^{14} \text {, Yang et al. }{ }^{15} \text { \& Saud } \\
\text { et al. }{ }^{17}\end{array}$ \\
\hline
\end{tabular}

\section{Conclusion}

Experimental studies about the relationship between cancer and the consumption of dietary polyphenol consumption gave different positive results. Majority of studies highlights their role in cancer prevention with the main focus on their anti-proliferative, anti-suppressive, anti-carcinogenesis, anti-oxidative, and anti-inflammatory and antiangiogenesis properties. Ingestion of polyphenols from fruits and vegetables also provide the strong protection against hormone related cancers especially colon, breast and prostate cancers. In future studies large clinical trials of polyphenols need to be conducted for assessing its higher validity and authenticity in cancer prevention.

\section{Acknowledgements}

None.

\section{Conflicts of interest}

Authors declare that there is none of the conflicts.

\section{References}

1. Ferlay J, Soerjomataram I, Ervik M, et al. Cancer Incidence and Mortality Worldwide. J Cancer. 2017;9:121-130.

2. (WHO) World Health Organization. Cancer.

3. IARC (International Agency for Research on Cancer). Global Cancer Statistics.

4. SKMCH (Shaukat Khanum Memorial Cancer Hospital). Cancer.

5. Spencer JP, Mohsen MM, Minihane AM, Mathers JC. Biomarkers of the Intake of Dietary Polyphenols: Strengths, Limitations and Application in nutrition research. Br J Nutr. 2016;99(1):12-22.

6. Li AN, Li S, Zhang Y, et al. Resources and Biological Activities of Natural Polyphenols. Nutrients. 2017;6(12):6020-6047.

7. Manach C, Mornad C, Jimenez L, et al. Polyphenols Food Sources and Bioavailability. Am J Clin Nutr. 2004;79(5):727-747.

8. Garcia VR, Nevedomskaya ER, Pancorbo A. Understanding the Metabolism of Polyphenols. Bioanal Chem. 2016;399:485-93.

9. Marzocchella L, Fantini M, Benvenuto M. Dietary Flavnoids Mechanism of action. Br J Nutr. 2016;5(3):200-210.

10. Deng GF, Lin X, Xu XR, et al. Antioxidant Capacities and Total Phenolic Contents of Fruits and Vegetables. J Funct Foods. 2017;6:260-266.
11. Scalbert A, Manach C, Remssy C. Dietary Polyphenols and the Prevention of Diseases. Anticancer Res. 2005;45(4):287-306.

12. Woo HD, Lee J, Choi IJ, et al. Dietary Flavonoids and Gastric Cancer. Nutr. 2014;6(11):4961-4973.

13. Korkina LG, Kostyuk VA, Pastore S. Plant polyphenols Mechanism against Cancer. Curr Med Chem. 2017;18:4011-4018.

14. Wang H, Zhang H, Tang L, et al. Resveratrol inhibits TGF-beta1-Induced Epithelial-to-Mesenchymal Transition and Suppresses Lung Cancer Invasion and Metastasis. Toxicol. 2013;303:139-146.

15. Yang Q, Wang B, Zang W, et al. Resveratrol Inhibits the Growth of Gastric Cancer by Inducing G1 phase arrest. J Cancer Res. 2015;10:615-622.

16. Wang Z, Li W, Meng X, et al. Resveratrol Induces Gastric Cancer Cell Apoptosis via Reactive Oxygen Species. Clin Exp Pharmacol Physiol. 2012;39(3):227-232.

17. Saud SM, Li W, Morris NL, et al. Resveratrol Prevents Tumorigenesis in Mouse Model of Kras Activated Sporadic Colorectal Cancer by Suppressing Kras. Carcinogen. 2014;35(12):2778-2786.

18. Aires V, Limagne E, Cotte AK, et al. Resveratrol Metabolites Inhibit Human Metastatic Colon Cancer Cells Progression and Synergize with Chemotherapeutic Drugs. Mol Nutr Food Res. 2013;57(7):1170-1181.

19. Rajasekaran D, Elavarasan J, Kumar A, et al. Resveratrol Interferes with $N$-nitrosodiethylamine-induced Hepatocellular Carcinoma at Early Stages in Male Rats. Mol Med Rep. 2015;4(6):1211-1217.

20. Arul D, Subramanian P. Naringenin Induces Growth Inhibition, Cell Cycle Arrest and Apoptosis in Human Hepatocellular Carcinoma. Pathol Oncol Res. 2017;19(4):763-770.

21. Song HM, Park GH, Eo HJ, et al. Naringenin-Mediated Expression Contributes to Apoptosis in Human Colon Cancer. Biomol Ther. 2016;24(2):140-146.

22. Lee CC, Liu KJ, Wu Y, et al. Sesamin Inhibits Macrophage-Induced Vascular Endothelial Growth Factor Expression and Proangiogenic Activity. Inflamm. 2016;34(3):209-221.

23. Kim HS, Wannatung T, Lee S, et al. Quercetin Enhances Hypoxiamediated Apoptosis via Direct Inhibition of AMPK Activity in Colon Cancer. Apoptosis. 2015;17(9):938-949.

24. Duo J, Ying GG, Wang GW, et al. Quercetin Inhibits Human Breast Cancer Cell Proliferation and Induces Apoptosis via Bcl-2. Mol Med Rep. 2014;5(6):1453-1456.

25. Zhao X, Wang Q, Yang S, et al. Quercetin Inhibits Angiogenesis in Human Breast Cancer. Eur J Pharmacol. 2016;781:60-68. 
26. Sharmila G, Athirai T, Kiruthiga B, et al. Chemopreventive Effect of Quercetin in Testosterone Induced Prostate Cancer. Nutr Cancer. 2015;66(1):38-46.

27. Jo E, Park S, Choi YS, et al. Kaempferol Suppresses Transforming Growth Factor-Beta1-Induced Epithelial-to-Mesenchymal Transition of Lung cancer. Neoplasia. 2015;17(7):525-537.

28. Song H, Bao J, Wei Y, et al. Kaempferol Inhibits Gastric Cancer Tumor Growth. Oncol Rep. 2016;33(2):868-874.
29. Azevedo C, Araujo JR, Guimaraes JT, et al. The Chemopreventive Effect of the Dietary Compound Kaempferol on the Human Breast Cancer by Inhibition of Glucose Cellular Uptake. Nutr Cancer. 2015;67(3):504-513.

30. Li C, Zhao Y, Yang D, et al. Inhibitory Effects of Kaempferol on the Invasion of Human Breast Carcinoma cells by Downregulating the Expression Metalloproteinase-9. Biochem Cell Biol. 2015;93(1):16-27. 